\title{
Méthodes statistiques pour la détermination de la distance d'arrêt maximale des avalanches
}

\author{
Ghali Adjel \\ CEMAGREF, Division Nivologie ou SMS/LMC/IMAG
}

Devant la difficulté d'utiliser un modèle numérique pour déterminer la distance d'arrêt maximale d'une avalanche et l'évaluation de sa période de retour, une approche statistique a vu le jour. Bien que celle-ci puisse apporter un élément de réponse à l'évaluation des périodes de retour, seule l'estimation de la distance d'arrêt maximale des avalanches fera l'objet de cet article. Deux méthodes statistiques ont été élaborées pour l'estimation de la distance d'arrêt maximale des avalanches. Elles sont issues de deux approches différentes des statistiques.

- La première approche, le plus souvent employée, est basée sur la régression d'un certain nombre de paramètres topographiques afin d'expliquer le paramètre décrivant l'étendue de l'avalanche maximale (avalanche dont la période de retour est supérieure à 100 ans). Cette méthode a été introduite par K. Lied et S. Bakkehøi [1] ; elle est communément appelée « méthode norvégienne » du fait de l'origine de ses créateurs.

- La seconde est, elle, basée sur l'identification de la loi du paramètre décrivant l'étendue de l'avalanche maximale. Cette méthode a été développée par D.M. McClung et K. Lied [2], qu'ils nomment souvent « méthode du rapport d'arrêt ".

Comme la CLPA contient l'enveloppe maximale des événements avalancheux recencés, ces deux méthodes peuvent être appliquées à un échantillon de 285 sites avalancheux de la CLPA de Haute-Tarentaise.

\section{MODÉLISATION PAR RÉGRESSION}

\subsection{Méthode norvégienne}

K. Lied et S. Bakkehøi [1] ont supposé qu'un site avalancheux pouvait se réduire à un profil en long. Ils y identifient les paramètres qu'ils jugent importants, comme l'indique la figure 1:

$\theta$ : angle de pente de la zone de rupture, mesuré sur carte entre deux lignes de niveau équidistantes de $100 \mathrm{~m}$, à partir $\mathrm{du}$ plus haut point de rupture.

$\beta$ : angle moyen du site du couloir d'avalanche, pris entre le plus haut point de rupture, le point du profil où la pente est de $10^{\circ}$ (ce point est, par extension, appelé point $\beta$ ) et l'horizontale ; on peut considérer qu'au-delà de ce point toutes les avalanches décélèrent.

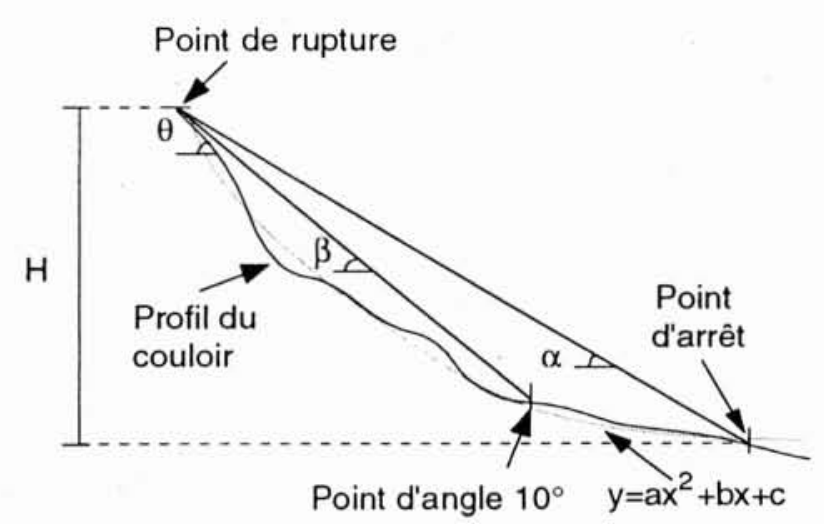

1. Paramètres d'un site avalancheux qui peut se réduire à un profil en long.

$H$ : dénivelée mesurée entre le point de rupture et le point le plus bas connu de la zone d'arrêt.

$y^{\prime \prime}$ : dérivée seconde du polynôme $y=a x^{2}+b x+c$ ajustant le profil du terrain.

A l'aide de ces paramètres, ils tentent de déterminer la relation entre $\alpha$ (l'angle entre le point de rupture et le point d'arrêt) et les autres paramètres afin d'obtenir un modèle de la forme $\hat{\alpha}=f\left(\theta, \beta, H, y^{\prime \prime}\right)$. De nombreux modèles, pour la plupart linéaires, ont été obtenus par K. Lied, S. Bakkehøi et par D.M. McClung. L'élaboration de modèles, suivant une classification paramétrique, a également été tentée par S. Bakkehøi et al. [3].

K. Lied et S. Bakkehøi [1] ont obtenu sur un échantillon de 111 sites avalancheux de Norvège, pour l'estimation de la distance maximale d'arrêt d'avalanches, les estimateurs suivants :

$$
\begin{aligned}
& \hat{\alpha}=0,97 \beta-1,4^{\circ} \\
& R=0,88 \text { et } \sigma=3,5^{\circ} \\
& \hat{\alpha}=\left(0,62-0,28 H y^{\prime \prime}\right) \beta+\left(0,19 H y^{\prime \prime}-2,3\right)^{\circ}+0,12 \theta \\
& R=0,95 \text { et } \sigma=2,3^{\circ}
\end{aligned}
$$

S. Bakkehøi et al. [3] obtient sur un échantillon de 206 sites avalancheux de Norvège, les estimateurs suivants : 


$$
\begin{aligned}
& \hat{\alpha}=0,96 \beta-1,4^{\circ} \\
& R=0,92 \text { et } \sigma=2,3^{\circ} \\
& \hat{\alpha}=0,92 \beta-7,9 \times 10^{-4}[H]+2,4 \times 10^{-4}[H] y^{\prime \prime} \theta+0,04^{\circ} \\
& R=0,92 \text { et } \sigma=2,28^{\circ}
\end{aligned}
$$

où $[H]$ est la dénivelée entre le point de rupture le plus haut et le point où la parabole ajustant le profil atteint son minimum.

Le premier estimateur doit être préféré au second, car il est plus simple tout en étant aussi précis.

Sur cet échantillon de 206 sites, il effectue également une classification suivant les paramètres topographiques $(\beta, H, \theta)$ et trouve :

Pour $\beta \leqslant 30^{\circ}$

$$
\begin{aligned}
& \hat{\alpha}=0,89 \beta+3,5 \times 10^{-2} \theta-2,2 \times 10^{-4}[H]-0,9^{\circ} \\
& R=0,84 \text { et } \sigma=1,49^{\circ}(N=68)
\end{aligned}
$$

Pour $30^{\circ}<\beta \leqslant 35^{\circ}$

$$
\begin{aligned}
& \hat{\alpha}=1,15 \beta-2,25 \times 10^{-3}[H]-5,9^{\circ} \\
& R=0,53 \text { et } \sigma=2,50^{\circ}(N=59)
\end{aligned}
$$

Pour $\beta>35^{\circ}$

$$
\begin{aligned}
& \hat{\alpha}=0.81 \beta+3,6 \times 10^{-2}[H] y^{\prime \prime} \theta+3,2^{\circ} \\
& R=0.62 \text { et } \sigma=2,67^{\circ}(N=79)
\end{aligned}
$$

Pour $H>900 \mathrm{~m}$ et $\beta \leqslant 30^{\circ}$

$$
\begin{aligned}
& \hat{\alpha}=0,81 \beta+0,035 \theta-2,6^{\circ} \\
& R=0,90 \text { et } \sigma=1,02^{\circ}(N=20)
\end{aligned}
$$

D.M. McClung et A.I. Mears [4] ont, eux, testé cette méthode sur plusieurs chaînes montagneuses de différentes régions :

Canada $\hat{\alpha}=0,93 \beta ; R^{2}=0,75 ; N=126$

Norvège $\hat{\alpha}=0,90 \beta ; R^{2}=0,87 ; N=127$

$$
\begin{array}{ll}
\text { Alaska } & \hat{\alpha}=0,74 \beta+3,67^{\circ} ; R^{2}=0,58 ; N=52 \\
\text { Colorado } & \hat{\alpha}=0,63 \beta+4,68^{\circ} ; R^{2}=0,50 ; N=130
\end{array}
$$$$
\text { Sierra Nevada } \hat{\alpha}=0,67 \beta+2,50^{\circ}: R^{2}=0,60 ; N=90
$$

$R$ est ici le coefficient de corrélation de Pearson (standard).

Pour les données du Canada et de la Norvège, ils montrent que l'ajout de paramètres topographiques n'améliore pas significativement l'estimation de $\alpha$.

\subsection{Application à la CLPA de Haute-Tarentaise}

Le CEMAGREF possède l'information spatiale concernant l'enveloppe maximale des avalanches «connnues à ce jour » sur les CLPA. Ces enveloppes sont gérées à l'aide du SIG (') (Système d'Information Géographique) ArcInfo. Après avoir choisi la CLPA de Haute-Tarentaise, nous avons sélectionné un nombre significatif de sites avalancheux. Pour cette zone d'étude, nous avons fait scannériser et vectoriser les courbes de niveaux au 1/25000, d'équidistance 10 mètres, par un prestataire de service. A l'aide des courbes de niveaux et des enveloppes maximales d'avalanches, il est possible de saisir des profils en long. Le SIG permet d'obtenir l'information tridimensionnelle de ces profils ; elle nous donnera la possibilité d'extraire de manière systématique les paramètres des diverses méthodes statistiques. Une sélection de 285 sites avalancheux a été effectuée; les profils en long ont été saisis sur ces sites. Un programme a également été conçu pour le calcul des différents paramètres afin d'automatiser le procédé.

Sur les 285 sites sélectionnés, on s'aperçoit que :

- pour $21 \%$ des sites, l'avalanche maximale n'atteint pas le point $\beta$,

- pour $19 \%$ des sites, l'avalanche maximale atteint plusieurs points $\beta$ (replats successifs),

- seulement $60 \%$ des sites répondent aux exigences du modèle norvégien.

Ces résultats sont comparables à ceux obtenus par [5]. Elle indique, en effet, qu'un certain nombre de sites ont des profils correspondant aux types suivants : rectilignes ne possédant pas de point $\beta$, trop irréguliers pour être ajustés correctement par un polynôme du second degré, avec replat intermédiaire... Ces profils ne correspondent pas à ceux définis par K. Lied et S. Bakkehøi.

En effectuant une régression sur les 168 sites répondant aux caractéristiques définies par K. Lied et S. Bakkehøi, avec $\beta$ pour variable explicative, on obtient :

$$
\begin{aligned}
& \hat{\alpha}=0,82 \beta+2,82^{\circ} \\
& R=0,81 \quad \text { et } \sigma=2,69^{\circ}
\end{aligned}
$$

En utilisant les autres paramètres topographiques $(\delta$ : angle entre le point de pente $10^{\circ}$ et le point d'arrêt, $y^{\prime \prime}, H$ ), les estimateurs suivants ont été trouvés:

$$
\begin{aligned}
& \hat{\alpha}=0,83 \beta+0,04 \delta+1,15^{\circ} \\
& R=0,85 \text { et } \sigma=2,54^{\circ}(N=125) \\
& \hat{\alpha}=0,92 \beta-3,3610^{3} y^{\prime \prime}+1,17^{\circ} \\
& R=0,84 \text { et } \sigma=2,47^{\circ}(N=168) \\
& \hat{\alpha}=0,95 \beta-3,6410^{3} y^{\prime \prime}+0,05 \delta+0,69^{\circ} \\
& R=0,88 \text { et } \sigma=2,26^{\circ}(N=125) \\
& \hat{\alpha}=0,82 \beta-4,6610^{3} y^{\prime \prime}+0,04 \delta+8,81 H y^{\prime \prime}+2,19^{\circ} \\
& R=0,89 \text { et } \sigma=2,21^{\circ}(N=125)
\end{aligned}
$$

(') «Base de données informatique dans laquelle les informations spatiales de type géographique sont traitées et analysées par l'intermédiaire de tables qui définissent les éléments graphiques et descriptifs » (DATA-IMAGE). 
En effectuant une classification suivant les valeurs des paramètres topographiques, on obtient :

$$
\begin{aligned}
& \text { suivant } H \\
& H \leqslant 500 \mathrm{~m} \\
& \hat{\alpha}=0,78 \beta+3,39^{\circ} \\
& R=0,77 \text { et } \sigma=3,07^{\circ}(N=104) \\
& H>500 \mathrm{~m} \\
& \hat{\alpha}=0,83 \beta+3,46^{\circ} \\
& R=0,91 \quad \text { et } \quad \sigma=1,34^{\circ}(N=64) \\
& \text { suivant } y^{\prime \prime} \\
& y^{\prime \prime} \leqslant 0,410^{3} \\
& \hat{\alpha}=0,97 \beta+1,06^{\circ} \\
& R=0,91 \text { et } \sigma=1,85^{\circ}(N=91) \\
& y^{\prime \prime}>0,410^{3} \\
& \hat{\alpha}=0,77 \beta+3,79^{\circ} \\
& R=0,72 \text { et } \sigma=3,27^{\circ}(N=77) \\
& H \leqslant 500 \mathrm{~m} \text { et } y^{\prime \prime} \leqslant 0,410^{3} \\
& \hat{\alpha}=0,84 \beta-2,08^{\circ} \\
& R=0,83 \text { et } \sigma=2,52^{\circ}(N=40) \\
& H \leqslant 500 \text { m et } y^{\prime \prime}>0,410^{3} \\
& \hat{\alpha}=0,72 \beta+5,06^{\circ} \\
& R=0,68 \text { et } \sigma=3,41^{\circ}(N=65) \\
& H>500 \mathrm{~m} \text { et } y^{\prime \prime}<0,410^{3} \\
& \hat{\alpha}=0,86 \beta+2,52^{\circ} \\
& R=0,91 \quad \text { et } \quad \sigma=1,41^{\circ}(N=52)
\end{aligned}
$$

suivant $\delta, H$

$$
\begin{aligned}
& 0^{\circ}<\delta \leqslant 10^{\circ} \\
& \hat{\alpha}=0,83 \beta+1,71^{\circ} \\
& R=0,86 \text { et } \sigma=2,46^{\circ}(N=106) \\
& 0^{\circ}<\delta \leqslant 10^{\circ} \text { et } H \leqslant 500 \mathrm{~m} \\
& \hat{\alpha}=0,76 \beta+2,89^{\circ} \\
& R=0,83 \text { et } \sigma=2,70^{\circ}(N=65) \\
& 0^{\circ}<\delta \leqslant 10^{\circ} \text { et } H>500 \mathrm{~m} \\
& \hat{\alpha}=0,86 \beta+2,06^{\circ} \\
& R=0,95 \text { et } \sigma=1,18^{\circ}(N=42)
\end{aligned}
$$

En effectuant une classification selon $\theta$ ou $\beta$, aucune amélioration n'a été obtenue pour l'estimation de $\alpha$. En conclusion, seuls $H, y^{\prime \prime}$ et $\delta$ permettent une amélioration des estimateurs lorsqu'on effectue une classification suivant leurs valeurs. En effectuant une classification croisée entre $H$ et $\delta$, on obtient, pour $0^{\circ}<\delta \leqslant 10^{\circ}$ et $H>500 \mathrm{~m}$, un estimateur précis.

\section{IIDENTIFICATION DE LA LOI DU PARAMÈTRE DESCRIPTIF}

\subsection{Méthode du rapport d'arrêt}

D.M. McClung et K. Lied [2] ont, eux, développé une approche différente de la précédente. Pour cela, ils définissent de nouveaux paramètres comme (fig. 2):

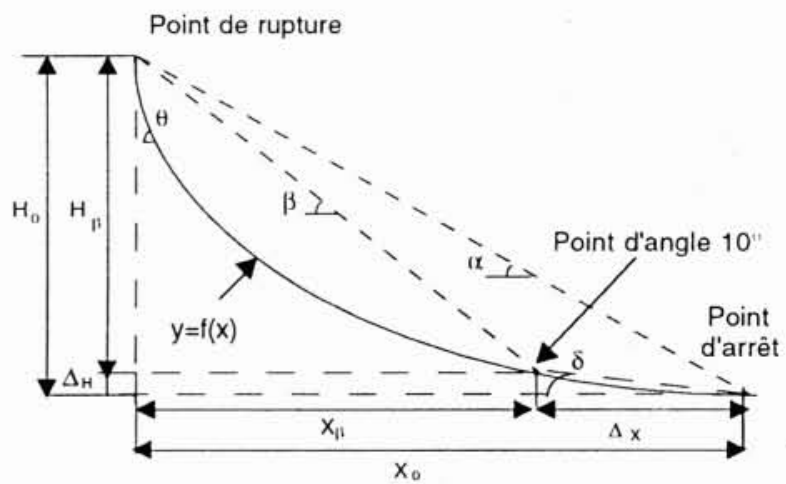

2. Paramètres d'un site avalancheux définis par D.M. McClung et $\mathbf{K}$. Lied.

$\Delta_{X}$ : distance horizontale ente le point $\beta$ (point où l'angle de la pente est égal à $10^{\circ}$ ) et le point d'arrêt.

$X_{\beta}$ : distance horizontale entre le point de rupture et le point $\beta$.

$\Delta_{H}$ : dénivelée entre le point d'angle $10^{\circ}$ et le point d'arrêt.

$H_{\beta}$ : dénivelée entre le point de rupture et le point d'angle $10^{\circ}$.

D.M. McClung et $\mathrm{K}$. Lied [2] montrent que $\Delta X / X_{\beta}$ est statistiquement indépendant, en première approximation de $\beta, \theta$ et $\delta$. Il est donc possible d'extrapoler sa valeur autour de sa moyenne. Le ratio $\Delta X / X_{\beta}$ n'étant pas une variable gaussienne, différentes distributions ont été testées, dont :

- log-normal

- Weibull et sa valeur extrême (Gumbel)

D.M. McClung et al. [4] montre que le rapport d'arrêt peut être ajusté par la distribution de Gumbel (ou loi des valeurs extrêmes). Pour simplifier les équations, il pose $x=\Delta x / X_{\beta}$. La densité de $x$ s'écrit alors : 


$$
\begin{aligned}
f(x)=\frac{1}{b} \exp [- & \left(\frac{x-u}{b}\right)- \\
& \left.-\exp \left(-\left[\frac{x-u}{b}\right]\right)\right]-\infty<x<+\infty
\end{aligned}
$$

où $b$ est le paramètre d'échelle et $u$ le paramètre de position.

Pour cette distribution, la moyenne est égale à $m=u+\gamma b$ (où $\gamma=0,57721 \ldots$, constante d'Euler) et la variance est égale à $\sigma^{2}=\left(\Pi^{2} / 6\right) b^{2}$. Le $p$-quantile (2) s'écrit :

$$
\begin{aligned}
& x_{p}=u-b \ln (-\ln p)=u+b Y_{p} \\
& p \text { est la probabilité de non-dépassement } \\
& Y_{p} \text { la variable centrée réduite }\left(^{3}\right)
\end{aligned}
$$

La probabilité de non-dépassement est alors :

$\operatorname{Pr}(x \leqslant x p)=\int_{-\infty}^{x_{p}} f(z) \mathrm{d} z=$

$$
\exp \left[-\exp \left(-\left[\frac{x p-u}{b}\right]\right)\right]
$$

D.M. McClung et al. [4] définit la probabilité de nondépassement, pour les $x_{i}$ rangés par ordre croissant $\left(x_{1}<\ldots<x_{N}\right)$, par:

$$
P_{i}=\frac{i-0,4}{N}
$$

D.M. McClung et A.I. Mears [6] obtiennent sur différentes chaînes montagneuses, les valeurs de $u$ et de $b$ suivantes (l'écart type, le coefficient de corrélation obtenus lors de la régression, le nombre des sites sélectionnés sont également donnés) :

\begin{tabular}{|l|c|c|c|c|r|}
\hline $\begin{array}{l}\text { Chaîne } \\
\text { montagneuse }\end{array}$ & $u$ & $b$ & $\sigma$ & $R^{2}$ & $N$ \\
\hline Canada & 0,079 & 0,070 & 0,012 & 0,98 & 79 \\
\hline Norvège & 0,143 & 0,077 & 0,011 & 0,98 & 80 \\
\hline Alaska & 0,185 & 0,108 & 0,023 & 0,97 & 52 \\
\hline Colorado & 0,288 & 0,202 & 0,040 & 0,98 & 130 \\
\hline Sierra Nevada & 0,374 & 0,206 & 0,041 & 0,98 & 90 \\
\hline
\end{tabular}

Les données de Norvège et du Canada ont été censurées pour $p \leqslant e^{-1}$.

\subsection{Application à la CLPA de haute-tarentaise}

Sur notre échantillon de sites, si l'on ne prend en compte que ceux atteignant un point $\beta$ et un seul, on obtient pour le rapport d'arrêt $\Delta x / X_{\beta}$ l'histogramme ci-contre (fig. 3):

On pose, comme l'a fait D.M. McClung [4], $x=\Delta x / X_{\beta}$. On obtient alors un échantillon de valeurs $x_{i}$ que l'on range par ordre croissant. On calcule, comme

$\left({ }^{2}\right)$ Valeur $x_{p}$ d'une variable aléatoire $x$ tel que $\operatorname{Pr}\left(x \leqslant x_{p}\right)=p$.

(3) Ici, variable aléatoire suivant la loi de Gumbel avec, comme paramètres, $u=0$ et $b=1$.

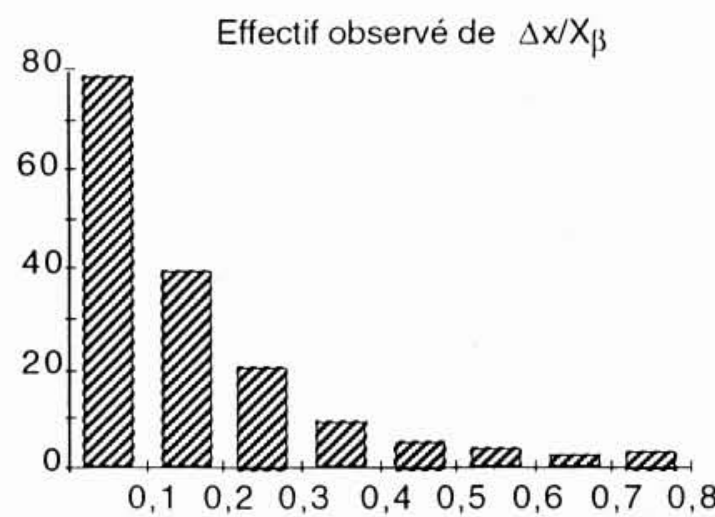

3. Histogramme du rapport d'arrêt $\Delta x / X_{\beta}$.

l'a fait D.M. McClung [4], à chaque $x_{i}$ une probabilité de non-dépassement à l'aide de l'équation (3). Le graphe des observations de $x$ pour notre échantillon est donné par la figure 4:

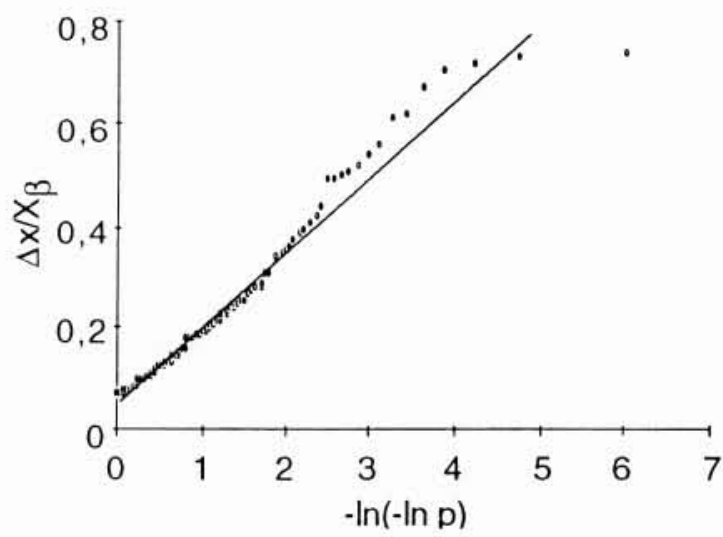

4. Graphe des observations du rapport d'arrêt.

En identifiant les paramètres $(u, b)$ de la loi des valeurs extrêmes, on obtient :

\begin{tabular}{|l|c|c|c|c|c|}
\hline $\begin{array}{l}\text { Chaîne } \\
\text { montagneuse }\end{array}$ & $u$ & $b$ & $\sigma$ & $R^{2}$ & $N$ \\
\hline Haute-Tarentaise & 0,052 & 0,150 & 0,031 & 0,97 & 106 \\
\hline
\end{tabular}

d'où

$$
x_{p}=0,052-0,150 \ln (-\ln p)
$$

Pour différentes valeurs de $p$ et à l'aide de l'équation (4) on peut calculer $x_{p}$ :

\begin{tabular}{|l|l|l|l|}
\hline$p$ & 0,5 & 0,9 & 0,99 \\
\hline$x_{p}$ & 0,10 & 0,39 & 0,75 \\
\hline
\end{tabular}


Le calcul d'une région de non-dépassement de probabilité $p$, à partir du point $\beta$, peut se calculer par la formule suivante :

$$
(\Delta x)_{p}=x_{p} X_{\beta}=[0,052-0,150 \ln (-\ln p)] X_{\beta}
$$

\section{III 口 CONCLUSION}

L'inconvénient majeur de ces deux méthodes statistiques est qu'elles postulent l'existence et l'unicité d'un point $\beta$ (point où la pente du couloir est égale à $10^{\circ}$ ). Or, dans l'échantillon des sites sélectionnés de la CLPA de HauteTarentaise, ce dernier n'existe pas toujours $(21 \%$ des avalanches n'atteignent pas ce point et $19 \%$ des sites possèdent plusieurs points $\beta$ ). Cette constatation est générale et peut être étendue aux Alpes et aux Pyrénées. Ces méthodes ne peuvent donc pas être appliquées à tous les sites avalancheux. D'autres méthodes ne faisant pas intervenir le point $\beta$ comme paramètre prépondérant, doivent être développées. Ces deux méthodes ont néanmoins l'avantage de pouvoir être facilement mises en œuvre dans un SIG. Elles permettent d'obtenir aisément, sur un profil en long choisi, le point extrême de l'avalanche maximale lorsque le point $\beta$ existe et est unique.

\section{Remerciements}

Je tiens à remercier l'ensemble de la Division Nivologie et tout particulièrement Laurent Buisson et Corinne Pietri pour les diverses suggestions et corrections qu'ils ont apportées à cet article.

\section{Bibliographie}

[1] LIED K. et BAKKEHøI S. - " Empirical calculations of snow-avalanche runout based on topographic parameters ". Journal of Glaciology, vol. 26, n 94, pp. 165-177, 1980.

[2] McClung D.M. et Lied K. - "Stastitical and geometrical definition of snow avalanche runout ", Cold Regions Science and Technology, vol. 13, pp. 107-119. 1987.

[3] BAKKeHøI S., DOMAAS U. et LiED K. - "Calculation of snow avalanche runout distance ", Annals of Glaciology, vol. 4, pp. 24-29, 1983.

[4] McClung D.M., Mears A.I. et Schaerer P. - « Extreme avalanche runout: Data from four mountain ranges ». Annals of Glaciology, vol. 13, pp. 180-184, 1989.

[5] BERTHIER B. - * Evaluation statistique des limites maximales atteintes par les avalanches à partir des données topographiques », document CEMAGREF, 1986.

[6] McClung D.M. et MEARS A.I. - * Extreme value prediction of snow avalanche runout ", Cold Regions Science and Technology, vol. 19, pp. 163-175, 1991. 